\title{
Temporomandibular Disorders in Children: A Mini Review
}

\section{Karoline de Melo Magalhães*, Bruna Caroline Tomé Barreto, Eduardo Otero Amaral Vargas and Ana Maria Bolognese}

Department of Pediatric Dentistry and Orthodontics, Universidade Federal do Rio de Janeiro, School of Dentistry, Rio de Janeiro, RJ, Brazil

*Corresponding Author: Karoline de Melo Magalhães, Department of Pediatric Dentistry and Orthodontics, Universidade Federal do Rio de Janeiro, School of Dentistry, Rio de Janeiro, RJ, Brazil.
Received: September 22, 2020

Published: November 28, 2020

(C) All rights are reserved by Benabdellah A., et al.

\begin{abstract}
Temporomandibular disorders (TMD) are dysfunctions that comprehend disorders of masticatory muscles, the temporomandibular joints and occlusion. They may be caused by macro or microtrauma, anatomical, genetic, systemic or psychological factors. Studies show that up to $30 \%$ of children and adolescents may present some type of TMD. Signs and symptoms of the condition may be diagnosed by questioning its history, palpating the painful areas, observing mouth opening and closing and performing analgesic blocking. Image exams such as panoramic radiograph, computed tomography, magnetic resonance and ultrasound may help this procedure. Possible treatments are patient education, physical therapy, behavioral therapy, medication prescription, occlusal splints, occlusal adjustment, orthodontic treatment, or even surgeries. A gold standard treatment has not been yet assessed, but considering the short age of patients, reversible procedures should be preferred when possible.
\end{abstract}

Keywords: Temporomandibular Disorders; Temporomandibular Joint Disorders; TMJ Disorders; Child

\section{Abbreviations}

TMJ: Temporomandibular Joint; TMD: Temporomandibular Disorders

\section{Introduction}

Temporomandibular disorders (TMD) are a subject of great relevance and discussion in Dentistry. They are characterized as a subgroup from craniofacial pain, involving the temporomandibular joint (TMJ), masticatory muscles and muscular and bony structures of the head and neck [1,2].

\section{Classification}

Okeson, in his book Management of Temporomandibular Disorders and Occlusion [3], divides the Temporomandibular disorders in three larger groups: functional disorders of the muscles, functional disorders of the temporomandibular joint and functional disorders of the dentition.
Muscle disorders may be regional myalgic disorders, such as myofascial pain (trigger point myalgia); and chronic muscle pain, which may be caused by perpetuating factors, centrally mediated myalgia or chronic systemic myalgic disorders (fibromyalgia).

Myofascial pain is characterized as very localized hypersensitive areas of painful stretched muscle bands on palpation [4]. These trigger points may produce, in most of times, referred pain in other regions rather than the trigger point location. In cases of chronic muscle pain caused by perpetuating factors, the causes may be local or systemic. Local factors are commonly characterized by not treated or improperly treated acute myalgic disorder or recurrent episodes of the same etiology with minimum periods of relief. On the other hand, prevalent systemic factors described are continued emotional stress, downregulation of the descending inhibitory system, sleep disturbances, learned behaviour, secondary gains due to pain and even depression [5]. 
Centrally mediated myalgia or persistent orofacial muscle pain is a continuous muscle pain disorder originating from Central Neural System effects felt peripherally, opposite of the neurogenic inflammation. Fibromyalgia, on the other hand, is characterized by a widespread musculoskeletal pain disorder in which tenderness is found in 11 or more of 18 specific tender point sites throughout the body [6].

Temporomandibular joint disorders may be subdivided in derangements of the condyle-disc complex, structural incompatibility of the articular surfaces, or inflammatory joint disorders. The firsts are represented by categories of increasing severity including: disc displacement with a single click (occurs only on the initial movement of mouth opening), disc displacement with a reciprocal clicking (occurs on the initial mouth opening movements and final mouth closening movement) and total disc displacement. The latter can lead to disc displacement with reduction or intermittent locking (when the person can resolve the disc displacement with no assistance) or to disc displacement without reduction or closed lock (when the person is unable to return the disc to its normal position and there is a restrainment of mouth opening) [7].

Possible structural incompatibilities are adherence, which is a temporary sticking of the articular surfaces, and the tearing apart is felt as a click; alterations in form or subluxation (hypermobility) and luxation (open lock). Inflammatory joint disorders include synovitis (inflammation on the synovial tissues), capsulitis (inflammation of the capsular ligament), retrodiscitis (inflammation of the retrodiscal tissues) and arthritides (destructive process of the condyle and fossa). Lastly, functional dentition disorders may appear as mobility of teeth, pulpitis or, most commonly, tooth wear [3].

\section{Etiological factors}

Since temporomandibular disorders have a multifactorial etiology, they arouse great interest in different specialties. Possible causes to joint derangements are macrotrauma, especially due to falling, but also resulting from vehicle or sports accidents; or microtrauma from parafunctional habits, such as bruxism, clenching and hyperextension. Predisposing factors as systemic (arthritis, systemic lupus erythematosus), hormonal (estrogen), genetic, anatomical (malocclusions, steep articular eminences, posture) and psychological (somatization, anxiety, obsessive-compulsive feelings, stress) may also be related [8,9]. In children, the greatest incidence is due to trauma, but there are also reports of syndromes or systemic disorders $[10,11]$.

\section{Epidemiology}

Epidemiological studies reported that $60 \%$ of the population and more than one third of children and adolescents present some type of TMD, and that severity and symptoms' prevalence are higher in girls between 12-15 years old and tend to persist in the course of time [12-14]. Also, dolicocephalic facial morphology has already been reported as related to TMD [15]. Some studies found TMD symptoms in $25 \%$ of children in their studied population [16] and others $[17,18]$ showed a prevalence of $23.7 \%$ and $34.7 \%$ in adolescents.

In a research in which students were evaluated, it was observed that among patients who presented some kind of disorder, $50.9 \%$ of them had mild, $21.8 \%$ moderate and $0.9 \%$ severe TMD. Also, among the ones who had moderate and severe TMD, 56\% of them presented some kind of alteration in head position [19]. Controversial results have been reported concerning the presence of symptoms in children and adolescents. While some claim that TMD is rare in the first dentition, others state that $34 \%$ of children in this phase present at least one symptom associated with TMD [20-23]. The same happens for mixed dentition, for which prevalence ranges from $5-10 \%[20,24]$, and permanent dentition, ranging from $5-32 \%[20,25,26]$.

\section{Diagnosis}

Seven major observations can assist in diagnosing temporomandibular disorders: history, mandibular restriction, mandibular interference, acute malocclusion, loading of the joint, functional manipulation, and diagnostic anesthetic blockade [3].

History is important for distinguishing muscular from joint disorders. Joint disorders are usually associated with a traumatic event, and muscular disorders are more related to emotional stress. Mouth opening restriction may be associated with both types of disorders. In intracapsular problems, the mouth cannot be opened wider, even if with mild passive force, representing a hard "end feel". On the other hand, in muscular disorders, mild passive force may increase mouth opening, representing a soft "end feel" [7].

Deviations or deflections of the mandible in mouth opening and closing are also signing of temporomandibular disorders. Deviations that occur at a certain point during opening and then return to midline, that alter with speed of opening, or that occur at the same point during opening and closing are likely to be caused by joint disorders. Deviations caused by muscular disorders are often 
large, inconsistent, sweeping movements not associated with joint sounds. As for deflections, if it is persistent on protrusion and restricted during contralateral movement, it's a sign of joint disorder, and if these signs are not present, we are dealing with a muscular disorder [27].

Acute malocclusion appears suddenly secondary to a disorder. The disorder may result in disocclusion or heavy contact in a specific area, depending on the affected tissues. Loading the joint is also a good predictive analysis for TMD, since manipulative forces do not produce pain in healthy joints. Besides, functional manipulation by having the patient bite a tongue blade may help locate the source of pain and differentiate muscular from intracapsular pain [28].

Regarding pain disorders, a possible diagnostic procedure, when the previous factors were not sufficient for diagnosis, is analgesic blocking, which can be of two types: muscle injections and nerve block injections. Muscle injections may be indicated on trigger points when suspecting pain referral. Nerve block injections are a possibility for identifying whether the painful structure is actually the source of pain. They could be dental blocks, auriculotemporal nerve block, or infraorbital nerve block [29].

Furthermore, TMJ imaging may be recommended. Panoramic radiograph is a good exam for evaluating condylar head morphology and angulation but does not permit evaluation of the joint space, soft tissues, or condylar motion. Cone Beam Computed Tomography can be used to detect bony abnormalities and fractures and to assess asymmetries. Magnetic resonance imaging (MRI) provides visualization of soft tissues, specifically the position and contours of the TMJ disc, and can be used to detect inflammation. Lastly, ultrasound is a noninvasive imaging method that allows viewing superficial lateral aspects of the TMJ $[30,31]$.

\section{Treatment}

It has not yet been assessed, on a long-term basis, the success of specific treatments for TMD in children, but it's been suggested that conservative and reversible therapies are effective in reducing TMD symptoms. Possible reversible therapies are: patient education, regarding the nature of the disorder and potential etiological factors; several types of physical therapy; behavioral therapy, as relaxation training, awareness of daytime clenching and bruxism; medication prescription; occlusal splints in order to provide orthopedic stability to the TMJ; and injections on the TMJ or trigger points, nerve blocks or acupuncture [32].
In some cases, irreversible therapies may also be needed, and those include: occlusal adjustment, orthodontic treatment or even surgeries such as orthognathic surgery, open joint TMJ surgery, and TMJ reconstruction. However, the indication of surgeries is mostly limited to cases of severe joint degeneration or tumor resection [33]. Nonetheless, for a definitive treatment, the clinician should aim to eliminate the etiology of the TMD or its consequence.

\section{Conclusion}

Temporomandibular disorders demand a vast process of investigation for its diagnosis due to its multifactorial etiology and multiple classification possibilities. A precise prevalence rate of this condition in children has not yet been assessed, but the existing literature shows a substantial value that must be taken into account. A gold standard treatment has not been assessed yet, but considering the short age of patients, reversible procedures should be preferred when possible.

\section{Acknowledgements}

The authors wish to thank Coordenação de Aperfeiçoamento de Pessoal de Nível Superior (CAPES), for supporting academic and professional development.

\section{Conflict of Interest}

We have no conflicts of interest to disclose.

\section{Bibliography}

1. Scrivani SJ., et al. "Temporomandibular disorders". The New England Journal of Medicine 359.25 (2008): 2693-2705.

2. Greene CS. "Diagnosis and treatment of temporo-mandibular disorders: emergence of a new care guidelines statement". Oral Surgery, Oral Medicine, Oral Pathology, Oral Radiology, and Endodontology 110.2 (2010): 137-139.

3. Okeson JP. "Management of Temporomandibular Disorders and Occlusion". 8th ed., Elsevier, (2020).

4. Fricton J. "Myofascial Pain: Mechanisms to Management". Oral and Maxillofacial Surgery Clinics of North America 28.3 (2016): 289-311.

5. Chisnoiu AM., et al. "Factors involved in the etiology of temporomandibular disorders - a literature review". Clujul Medical 88.4 (2015): 473-478.

6. Bellato, E., et al. "Fibromyalgia syndrome: etiology, pathogenesis, diagnosis, and treatment". Pain Research and Treatment 426130 (2012): 1-17. 
7. deLeeuw R., et al. "Orofacial Pain: Guidelines, Assessment, Diagnosis, and Management". 6th ed., Quintessence Publishing (2018).

8. Venkateswaran S., et al. "Diagnosis and Management of Temporomandibular Joint Disorders- What the Medical and Dental practitioners should know?" Chettinad Health City Medical Journal. 2.2 (2013): 52-59.

9. Motghare V., et al. "Association Between Harmful Oral Habits and Sign and Symptoms of Temporomandibular Joint Disorders Among Adolescents". Journal of Clinical and Diagnostic Research 9.8 (2015): ZC45-ZC48.

10. Gray AR and Geoffrey R Barker. "Idiopathic blepharospasmoromandibular dystonia syndrome (Meige's syndrome) presenting as chronic temporomandibular joint dislocation". British Journal of Oral and Maxillofacial Surgery 29 (1991): 97-99.

11. Ludovic S., et al. "Bilateral dislocation of the Temporomandibular Joint in children". Journal of Oral and Maxillofacial Surgery 76.11 (2018): 2307-2315.

12. Egermark-Eriksson I., et al. "A longitudinal study on malocclusion in relation to signs and symptoms of cranio-mandibular disorders in children and adolescents". European Journal of Orthodontics 12 (1990): 399-407.

13. Magnusson T., et al. "Changes in subjective symptoms of craniomandibular disorders in children and adolescents during a 10-year period". Journal of Oral and Facial Pain and Headache 7 (1993): 76-82.

14. Pilley JR., et al. "A survey of craniomandibular disordes in 500 19-year-olds". European Journal of Orthodontics 19 (1997): $57-$ 70.

15. Wolford LM. "Idiopathic condylar resorption of the temporomandibular joint in teenage girls (cheerleaders syndrome)". Baylor University Medical Center Proceedings 14.3 (201): 246252.

16. Bilgiç F and İbrahim Erhan Gelgör. "Prevalence of Temporomandibular Dysfunction and its Association with Malocclusion in Children: An Epidemiologic Study". The Journal of Clinical Pediatric Dentistry 41.2 (2017): 161-165.

17. Ebrahimi M., et al. "Temporomandibular disorders and related factors in a group of Iranian adolescents: a cross-sectional survey". Journal of Dental Research, Dental Clinics, Dental Prospects 5 (2011): 123-127.
18. Tecco S., et al. "Signs and symptoms of temporomandibular joint disorders in Caucasian children and adolescents". Cranio 29.1 (2011): 71-79.

19. Chaves PJ., et al. "Incidence of postural changes and temporomandibular disorders in students". Acta Ortopedica Brasileira 25.4 (2017).

20. American Academy of Pediatric Dentistry. "Acquired temporomandibular disorders in infants, children, and adolescents". Pediatric Dentistry 37.5 (2015): 78-84.

21. Nydell A., et al. "Craniomandibular disorders in children-a critical review of the literature". Swedish Dental Journal 18.5 (1994): 191-205.

22. Bonjardim LR., et al. "Signs and symptoms of temporomandibular joint dysfunction in children with primary dentition". Journal of Clinical Pediatric Dentistry 28.1 (2003):53-58.

23. Kohler AA., et al. "Prevalence of symptoms and signs indicative of temporomandibular disorders in children and adolescents. A cross-sectional epidemiological investigation covering two decades". European Archives of Paediatric Dentistry 10.1 (2009): 16-25.

24. Thilander B., et al. "Prevalence of temporomandibular dysfunction and its association with malocclusion in children and adolescents: an epidemiologic study related to specified stages of dental development". The Angle Orthodontist 72.2 (2002): 146-154.

25. Nilsson IM., et al. "Impact of temporomandibular disorder pain in adolescents: differences by age and gender". Journal of Oral and Facial Pain and Headache 23.2 (2009): 115-122.

26. Nilsson IM., et al. "Headache and comorbid pains associated with TMD in adolescents". Journal of Dental Research 92.9 (2013): 802-807.

27. Nowak AJ., et al. "Pediatric Dentistry Infancy through Adolescence". 6th ed., Elsevier, (2019).

28. McNamara Jr JA., et al. "Occlusion, orthodontic treatment, and temporomandibular disorders: a review". Journal of Oral and Facial Pain and Headache 9 (1995): 73-90.

29. Vazquez-Delgado E., et al. "Myofascial pain associated to trigger points: a literature review. Part 2: differential diagnosis and treatment". Medicina Oral Patologia Oral y Cirugia Bucal 15.4 (2010): e639-e643. 
30. DeSenna BR., et al. "Imaging diagnosis of the temporomandibular joint: Critical review of indications and new perspectives". Oral Radiology 25.2 (2009): 86-98.

31. Hunter A and Sajitha Kalathingal. "Diagnostic imaging for temporo- mandibular disorders and orofacial pain". Dental Clinics of North America 57.3 (2013): 405-418.

32. List T and Stefan Axelsson. "Management of TMD: Evidence from systematic review and meta-analysis". Journal of Oral Rehabilitation 37.6 (2010): 430-451.

33. Choinard AF., et al. "Acquired abnormalities of the TMJ". Oral and Maxillofacial Surgery Clinics of North America 30.1 (2018): 83-96.

\section{Assets from publication with us}

- Prompt Acknowledgement after receiving the article

- Thorough Double blinded peer review

- Rapid Publication

- Issue of Publication Certificate

- High visibility of your Published work

Website: www.actascientific.com/

Submit Article: www.actascientific.com/submission.php Email us: editor@actascientific.com

Contact us: +919182824667 